\begin{tabular}{|c|l|}
\hline Title & $\begin{array}{l}\text { Detailed investigation of thermal convection in a liquid metal under a horizontal magnetic field: Suppression of } \\
\text { oscillatory flow observed by velocity profiles }\end{array}$ \\
\hline Author(s) & $\begin{array}{l}\text { Y anagisawa, Takatoshi; Y Yamagishi, Y asuko; Hamano, Y ozo; Tasaka, Y uji; Y ano, Kanako; Takahashi, Jumpei; Takeda, } \\
\text { Y asushi }\end{array}$ \\
\hline Citation & $\begin{array}{l}\text { Physical Review E, 82(5), 056306 } \\
\text { https://doi.org/10.1103/PhysRevE.82.056306 }\end{array}$ \\
\hline Issue Date & 2010-11 \\
\hline Doc URL & http://hdl.handle.net/2115/44394 \\
\hline Rights & O2010 The A merican Physical Society \\
\hline Type & article \\
\hline File Information & PRE82-5_056306.pdf \\
\hline
\end{tabular}

Instructions for use 


\title{
Detailed investigation of thermal convection in a liquid metal under a horizontal magnetic field: Suppression of oscillatory flow observed by velocity profiles
}

\author{
Takatoshi Yanagisawa, ${ }^{*}$ Yasuko Yamagishi, and Yozo Hamano \\ Institute for Research on Earth Evolution, Japan Agency for Marine-Earth Science and Technology, Yokosuka, Japan \\ Yuji Tasaka, Kanako Yano, Jumpei Takahashi, and Yasushi Takeda \\ Graduate School of Engineering, Hokkaido University, Sapporo, Japan
}

(Received 8 July 2010; revised manuscript received 1 September 2010; published 10 November 2010)

\begin{abstract}
Thermal convection experiments in a liquid gallium layer were carried out with various intensities of uniform horizontal magnetic fields. The gallium layer was in a rectangular vessel with a 4:1:1 length ratio (1 is the height), where the magnetic field is applied in the direction normal to the longest vertical wall. An ultrasonic velocity profiling method was used to visualize the spatiotemporal variations in the flow pattern, and the temperature fluctuations in the gallium layer were also monitored. The observed flow pattern without a magnetic field shows oscillating rolls with axes normal to the longest vertical wall of the vessel. The oscillatory motion of the flow pattern was suppressed when increasing the applied magnetic field. The flow behavior was characterized by the fluctuation amplitude of the oscillation and the frequency in the range of Rayleigh numbers from $9.3 \times 10^{3}$ to $3.5 \times 10^{5}$ and Chandrasekhar numbers 0-1900. The effect of the horizontal magnetic field on the flow pattern may be summarized into three regimes with increases in the magnetic intensity: (1) no effect of the magnetic field, (2) a decrease in the oscillation of the roll structure, and (3) a steady two-dimensional roll structure with no oscillation. These regimes may be explained as a result of an increase in the dominance of Lorentz forces over inertial forces. The power spectrum from the temperature time series showed the presence of a convective-inertial subrange above Rayleigh numbers of $7 \times 10^{4}$, which suggests that turbulence has developed, and such a subrange was commonly observed above this Rayleigh number even with applied magnetic fields when the rolls oscillate.
\end{abstract}

DOI: 10.1103/PhysRevE.82.056306

PACS number(s): 47.20.Bp, 47.27.De, 47.65.-d

\section{INTRODUCTION}

Rayleigh-Bénard convection in liquid metals under the influence of an external magnetic field is an important problem in fluid dynamics. Especially, to clarify the characteristics of turbulence affected by magnetic fields is important for engineering and geoscience applications. For example, magnetic fields are applied to suppress turbulence in liquid metals in tanks to enable stable crystal growth. The flow of liquid iron in the outer core of the earth may be turbulent because of its very large size, and the characteristics of the turbulence may be controlled by the geomagnetic field. Liquid metals are characterized by low viscosity, high thermal conductivity (low Prandtl number), and high electric conductivity. Electric currents are induced in the liquid metal flowing in the magnetic field, which generates Lorentz forces. The Lorentz force in turn deforms the flow behavior, making the flow very different from nonmagnetic field situations.

A Rayleigh-Bénard convection system is described by the Rayleigh number $\left(\mathrm{Ra}=\alpha g \Delta T L^{3} / \kappa \nu\right)$ and the Prandtl number $(\operatorname{Pr}=\nu / \kappa)$, where $\alpha$ is the volume coefficient of thermal expansion, $g$ is the gravitational acceleration, $\Delta T$ is the vertical temperature difference across the fluid layer, $L$ is the layer thickness, $\kappa$ is the thermal diffusivity, and $\nu$ is the kinematic viscosity. In general, the Pr of liquid metals is on the order of $10^{-2}$, which means that thermal diffusion is much larger than momentum diffusion. Additional to $\mathrm{Ra}$ and $\mathrm{Pr}$, the effect of

\footnotetext{
*yanagi@jamstec.go.jp
}

the applied magnetic field is quantified by the Chandrasekhar number $\left(Q=B^{2} L^{2} \sigma / \rho \nu\right)$ or the Hartmann number (Ha $\left.=B L \sqrt{\sigma / \rho \nu}=Q^{1 / 2}\right)$ that represents the ratio of the magnetic force to the viscous force, where $\sigma$ is the electrical conductivity, $\rho$ is the fluid density, and $B$ is the intensity of the applied magnetic field. The magnetic Reynolds number $(\mathrm{Rm}=\mu \sigma U L)$ represents the intensity ratio of the induced magnetic field to the applied magnetic field, and here $\mu$ is the magnetic permeability, with $U$ as the representative velocity of the flow. The Rm is expressed by the product of the Reynolds number $(\operatorname{Re}=U L / \nu)$ and the magnetic Prandtl number $(\mathrm{Pm}=\nu / \eta)$, where $\eta$ is the magnetic diffusivity of the fluid expressed by $\eta=1 / \nu \sigma$. The Pm of liquid metals is on the order of $10^{-6}$, which means that the magnetic field is highly diffusive when compared to the momentum diffusion. For thermal convection of liquid metals in laboratory scales, Re is below $10^{4}$, making $\mathrm{Rm}$ small and the effect of an induced magnetic field by the fluid motion is negligible.

In general, magnetic fields stabilize the convective flow of liquid metals depending on the direction and intensity of the field (e.g., [1-5]). In the Rayleigh-Bénard setting, theoretical studies indicate that a vertical magnetic field delays the onset of convective motion and raises the critical $\mathrm{Ra}$ where convection is initiated. Horizontal magnetic fields however do not raise the critical Ra, but align the axes of convective rolls along the field direction (see [6]). Just above the critical Ra, thermal convection of low-Pr fluids like liquid metals becomes time dependent, and there are oscillatory instabilities such as "traveling-wave convection" in the direction of the roll axis (e.g., $[7,8])$. The onset of oscillatory convection is 
affected both by vertical [9] and horizontal [10] magnetic fields. This delay of the onset of oscillatory convection has been confirmed by laboratory experiments (e.g., [11-16]). Libchaber et al. [11] investigated thermal convection of mercury under a horizontal magnetic field by measuring temperature fluctuations, and reported that there are increases in the transition $\mathrm{Ra}$ where oscillatory convection is initiated with increases in $Q$ values. Chiffaudel et al. [13] also used mercury to study oscillating convection patterns and frequencies depending on $\mathrm{Ra}$ in the presence of horizontal magnetic fields. They reported hysteresis in the oscillation behavior at increases and decreases in Ra.

At intermediate $\mathrm{Ra}$ values $\left(10^{4} \leq \mathrm{Ra} \leq 10^{5}\right)$ where the flow may be turbulent in the absence of magnetic field, the effect of the applied magnetic field is thought to suppress turbulence and to produce a nonisotropic flow structure. Theoretical studies in this range of $\mathrm{Ra}$ values are difficult because of strong nonlinearities, making experimental investigations here more important. Burr and Müller [16] used a sodium potassium alloy and studied the effect of horizontal magnetic fields on the convective flow for $10^{3} \leq \mathrm{Ra} \leq 10^{5}$ and $0 \leq Q \leq 10^{6}$, by measuring the heat transport (Nusselt number) and monitoring the anisotropy of the flow with a four-element temperature probe. The results showed increases in anisotropy in the convection flow with increases in $Q$.

These experimental studies elucidated aspects of the effect of magnetic fields on the suppression of oscillatory convection. However, the global structure of the flows and the transition to turbulence in liquid metal convection both with and without magnetic fields are not fully understood, mainly because of little data on convection patterns and flow velocities. Liquid metals are opaque fluids, and optical methods for flow measurements cannot be applied to observe the internal flows. As noted above, most experimental studies of liquid metal convection have focused on the temperature fluctuations in the fluid layer or measurements of the Nusselt numbers. By using a "transparent" water solution of electrolyte and a superconducting magnet, Andreev et al. [17] succeeded in visualizing the flow pattern under the influence of intense magnetic fields $(\sim 10 \mathrm{~T})$. They observed quasi-twodimensional convection patterns in the direction of the magnetic field and noted the increase in heat transfer, but the Pr of the fluid was $\sim 10$, and the behavior may be different from liquid metals. Hence, measurements of velocity profiles in liquid metals are expected, and it could enable a better understanding of the characteristics of the flow pattern and turbulence in liquid metals.

Direct measurements of the spatiotemporal flow field of thermal convection in liquid metals have been realized in recent years by the ultrasonic velocity profiler (UVP) method. With UVP, it is possible to measure flow velocity profiles along ultrasonic beam lines in real time by the Doppler effect (see [18]). Applications of UVP to RayleighBénard convection with liquid metals have shown flow patterns with fluctuations [19-22]. A report by Yanagisawa et al. [22] has used a 5:1.25:1 geometry (with a height of $40 \mathrm{~mm}$ ) vessel and visualized the flow pattern in a gallium layer without an applied magnetic field by simultaneous measurements of the velocity profile and temperature fluctuations for moderate Ra values $\left(1 \times 10^{4} \leq \mathrm{Ra} \leq 2 \times 10^{5}\right)$. Here, the presence of a roll-like structure with oscillatory behavior, even in the Ra range where the temperature power spectrum shows features of developed turbulence $\left(\mathrm{Ra} \geq 10^{5}\right)$, was reported. The flow structure was interpreted as a continuously developed one from the oscillatory instability of two-dimensional roll convection around the critical $\mathrm{Ra}$. It was showed that both the velocity of the flows and the frequency of the oscillation increase proportional to the square root of $\mathrm{Ra}$, and that the oscillation time of the roll structure is comparable to the time to complete one circulation of the flow.

This paper is a further investigation of the effects of applied horizontal magnetic fields on the behavior of convective flows of liquid gallium in a similar vessel. The aim of the present study is (a) to visualize the flow pattern and suppression process of fluctuating convection with increases in the horizontal magnetic field, (b) to classify the regimes of flow in relation to $\mathrm{Ra}$ and $Q$, and (c) to quantify features of the turbulence in the magnetic fields. The target range of $\mathrm{Ra}$ values is $9.3 \times 10^{3}-3.5 \times 10^{5}$, from about 5 to 200 times the critical $\mathrm{Ra}(=1708)$, and these are attained by direct measurement of flow velocity profiles with simultaneous measurements of temperature fluctuations in the gallium layer.

\section{APPARATUS AND METHODS}

Figure 1 shows the vessel for the liquid metal convection and the Helmholtz coil system with the positions of the various measurement points used in this study. Liquid gallium was used as the working fluid, and the sidewalls of the vessel are 10-mm-thick glass, with electric conductivity much lower than gallium and will be considered as a perfect insulator. The top and bottom plates are made of copper, and the temperature of each plate is maintained by circulating water. The inside measurements of the vessel are $200 \mathrm{~mm}$ long, 50 $\mathrm{mm}$ wide, and $50 \mathrm{~mm}$ high, giving an aspect ratio of 4 (length to height). A Helmholtz coil system is used to apply uniform magnetic fields to the vessel. The horizontal magnetic field was applied by a controllable dc power source, with intensity $B_{h}$ from 0 to $19 \mathrm{mT}$. The diameter of the coil is $600 \mathrm{~mm}$, and the spatial variance in the magnetic field is assured to be small, within $2 \%$ around the position of the vessel. The direction of the applied horizontal magnetic field in this paper is perpendicular to the long side of the vessel as shown in Fig. 1(b). The three components of the magnetic field were monitored outside the vessel by a magnetometer; the intensity of the vertical component of the ambient magnetic field is $0.04 \mathrm{mT}$ around the vessel. A comparison of the flow behavior in the ambient field and in a magnetic shielded box with intensity below $0.001 \mathrm{mT}$ confirmed that the ambient field has no effect on the flow. The range of Chandrasekhar numbers $(Q)$ realized in this system is from 0 to $1.9 \times 10^{3}$, which corresponds to Hartmann numbers (Ha) from 0 to 44 . A UVP was used to measure the velocity field of the flow in the liquid metal. The lines of measurement for the flow velocity were set along the long horizontal direction of the vessel [numbers 1-3 in Fig. 1(a)], and the projected component of the flow velocity on each line was measured. Further details of the experimental procedure with liquid gal- 

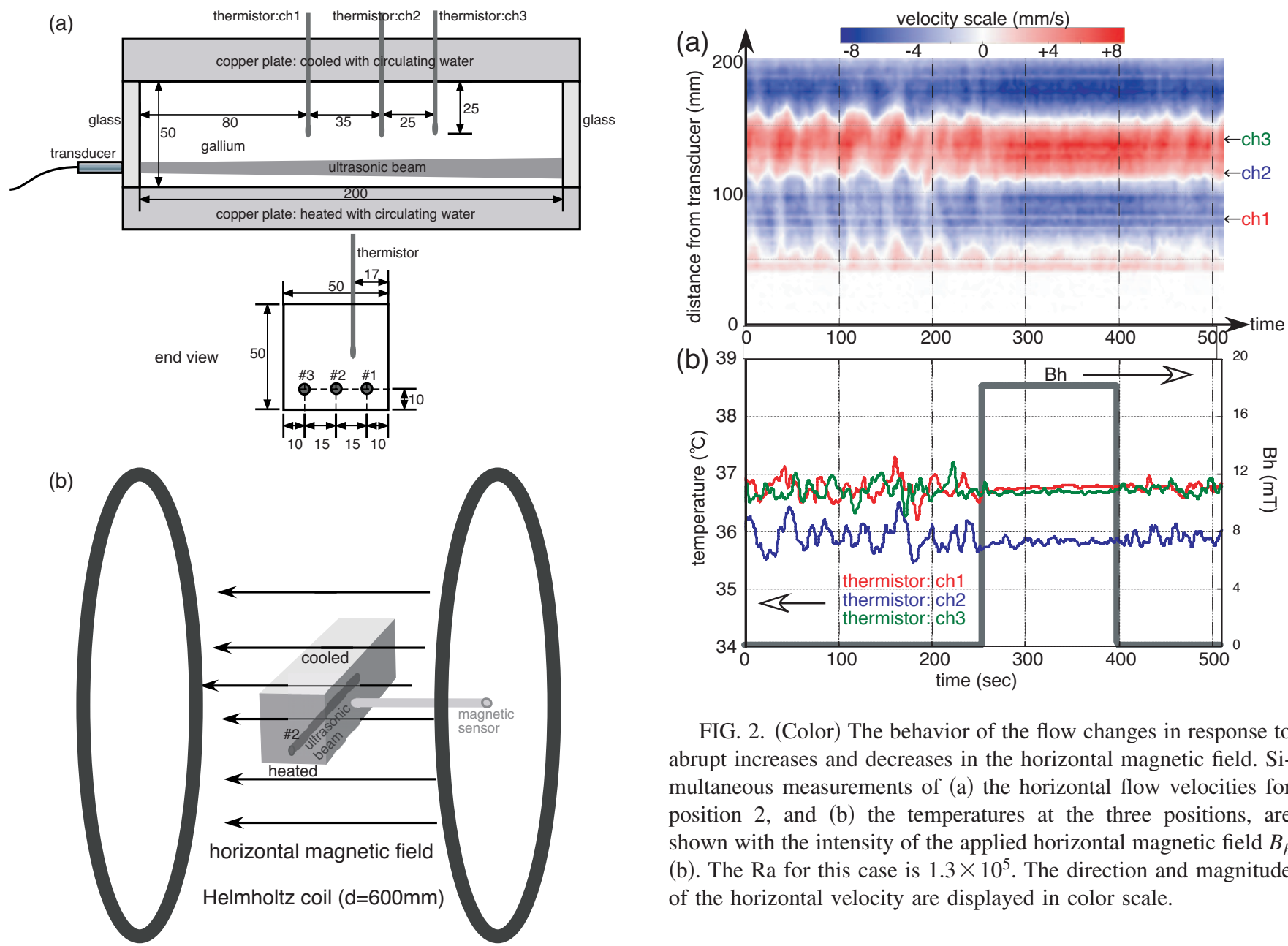

FIG. 2. (Color) The behavior of the flow changes in response to abrupt increases and decreases in the horizontal magnetic field. Simultaneous measurements of (a) the horizontal flow velocities for position 2, and (b) the temperatures at the three positions, are shown with the intensity of the applied horizontal magnetic field $B_{h}$ (b). The Ra for this case is $1.3 \times 10^{5}$. The direction and magnitude of the horizontal velocity are displayed in color scale.

FIG. 1. (Color online) (a) The top is a side view of the vessel used in the experiments, and the bottom is an end view. The numbers show the dimensions in mm. Numbers 1-3 are the positions of the ultrasonic transducers, and ch1-ch3 are the positions of the thermistor probes. (b) Schematic view of the Helmholtz coil system and the vessel in the horizontal magnetic field.

lium are provided elsewhere [21]. The melting temperature of gallium is $29.8^{\circ} \mathrm{C}$, and the temperature of the circulating water for cooling the top plate was maintained at $32.0^{\circ} \mathrm{C}$, with the temperature of the bottom plate set to vary the Rayleigh number values, $\mathrm{Ra}$. The temperature fluctuations in the gallium layer were measured by thermistors at three points [ch1-ch3 in Fig. 1(a)]. The calculations of Ra and $Q$ used the material properties of gallium summarized in [23], where the Prandtl number (Pr) of gallium around this temperature is 0.025 , and the magnetic Prandtl number $(\mathrm{Pm})$ is $1.5 \times 10^{-6}$.

\section{RESULTS}

\section{A. Suppression of oscillations in the flow by a horizontal magnetic field}

The following describes the visualization of the flow pattern associated with abrupt changes in an applied magnetic field $B_{h}$. Figure 2 shows the behavior of the flow associated with an instantaneous increase and decrease in the horizontal

magnetic field. In this experiment, Ra was $1.3 \times 10^{5}$. Temporal variations of the flow pattern at position 2 are shown in Fig. 2(a), and temperature fluctuations at channels 1-3 are shown in Fig. 2(b) with the intensity of horizontal magnetic field monitored by a magnetometer. The vertical axis in Fig. 2(a) shows the position in the vessel, with $0 \mathrm{~mm}$ the transducer end and $200 \mathrm{~mm}$ the opposite end of the vessel. The direction and magnitude of the horizontal velocity are shown in color, blue (minus) shows a flow toward the transducer and red (plus) flow away from the transducer. The left vertical axis in Fig. 2(b) shows the temperature in centigrade, and the right axis is the intensity of the applied horizontal magnetic field in millitesla. The horizontal axes show the time elapsed in the experiment.

From 0 to $250 \mathrm{~s}$, the applied magnetic field is zero and four oscillating rolls of convection are observed [schematically suggested in Figs. 3(a-1) and 3(a-2)]. The oscillatory motion of rolls generates smaller scale eddies (turbulence), which is indicated in the observed temperature fluctuations. The motion of rolls is not a purely periodic oscillation but having variation of the period (30-40 s). The oscillating rolls are typically observed for this rectangular vessel [22]. This oscillating four-roll pattern involving turbulence is a continuously developed structure from the oscillatory instability emerged just above the critical Ra, and the dominant period of its oscillation is comparable to the circulation time of the flow. 

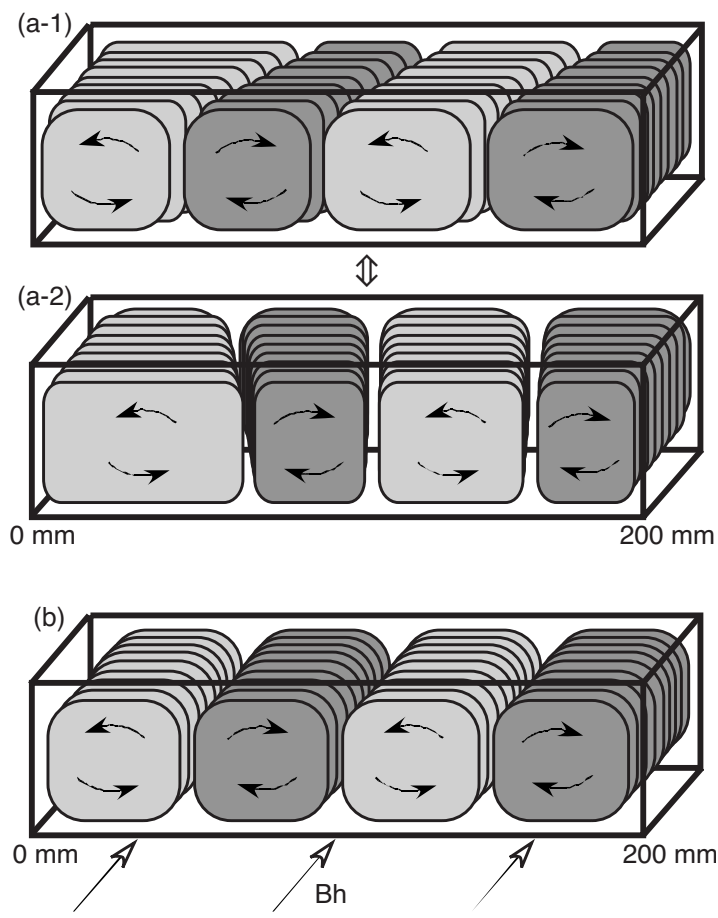

FIG. 3. Schematic representation of the three-dimensional flow structure inferred from the velocity profiles. The results showed four convection rolls both with and without an applied magnetic field. (a) Convection rolls oscillate like (a-1) and (a-2) patterns in the absence of an applied magnetic field. (b) Under a strong horizontal magnetic field, an almost steady state with the axes fixed in the direction of the magnetic field is established.

The white stripes in Fig. 2(a) (velocity=0) correspond to the boundaries between rolls. The central downwelling position fluctuates from 105 to $115 \mathrm{~mm}$ from the transducer end, corresponding to about one fifth of a roll width $(\sim 50 \mathrm{~mm})$. The velocity data in the positions between 0 to $40 \mathrm{~mm}$ are not clearly measurable because of the near-field effect of the ultrasonic measurements. These velocity measurements indicate that the ch1 probe is located in the second roll from the transducer sidewall, ch2 is at the central downwelling region between the second and the third rolls, and ch3 is in the third roll. The mean value of the temperature at ch2 is lower than at ch1 and ch3, as it is at a downwelling region between rolls. The timing and amplitude of the temperature fluctuations correlate well with the fluctuation in the flow pattern recorded by the UVP, and indicate that the oscillatory behavior of the flow induces temperature fluctuations.

At $250 \mathrm{~s}$ in Fig. 2, a horizontal magnetic field of $B_{h}$ $=18 \mathrm{mT}$ was applied. The velocity measurements show that, with a time lag about $30 \mathrm{~s}$, the oscillation of the flow pattern disappeared and the flow assumed an almost twodimensional steady state. Here, the amplitude of the temperature fluctuations for ch1 and ch3 decreased to about $10 \%$ of those without magnetic field, and to $15 \%$ for $\operatorname{ch} 2$. This indicates that the axis of the roll structure is aligned in the direction of the applied magnetic field and that an almost steady state of flow is realized [schematic view in Fig. 3(b)].

At $400 \mathrm{~s}$, the intensity of the applied magnetic field was reduced to zero. Following this the oscillation of the roll structure was reestablished, but both the range of the pattern oscillation and the amplitude of the temperature fluctuations were smaller than for the $0-250 \mathrm{~s}$ period. The oscillating range of central downwelling region was about half, and the amplitudes of the temperature fluctuations at all these channels were about $60 \%$.

In summary, this experiment shows that a horizontal magnetic field affects the flow pattern, and that the time scale of the flow to adjust to a new magnetic field is comparable to the circulation time of the rolls of the flow. Additionally, there is hysteresis in the oscillation amplitude between the increases and decreases in the magnetic field. The amplitude of the fluctuations tends to be smaller when the intensity of the magnetic field is decreased. In the following, the experiments will be limited to increases in the magnetic field.

\section{B. Effect of stepwise increases in the intensity of a horizontal magnetic field at fixed Ra values}

The behavior of the flow is affected by the magnetic field, and a systematic investigation of the effect of a horizontal magnetic field $\left(B_{h}\right)$ on the convective flow properties was made with six $\mathrm{Ra}$ values. The vertical temperature differences in the gallium layer were $0.2,0.5,0.9,1.7,3.1$, and 8.7 $\mathrm{K}$, corresponding to $\mathrm{Ra}$ values of $9.3 \times 10^{3}, 1.9 \times 10^{4}, 3.7$ $\times 10^{4}, 7.1 \times 10^{4}, 1.3 \times 10^{5}$, and $3.5 \times 10^{5}$, respectively. After establishing the thermally balanced state of convection at each $\mathrm{Ra}$ with no magnetic field, the intensity of the horizontal magnetic field was increased stepwise from $B_{h}=0 \mathrm{mT}$. At each magnetic field, after waiting more than $100 \mathrm{~s}$ for the flow behavior to stabilize, the measurements of the flow velocity by the UVP at positions 1 and 3 in Fig. 1 and the temperature by all three thermistor probes were acquired. A basic flow structure with four rolls and downwelling at the center and both sidewalls was observed throughout the series of experiments at all $\mathrm{Ra}$ values and all magnetic intensities in this vessel.

Figure 4 shows the results of the velocity measurements for six intensities of the horizontal magnetic field. The variations in the flow patterns are for $\mathrm{Ra}=3.7 \times 10^{4}$ (left) and $1.3 \times 10^{5}$ (right) at position 1 . The color scale of the velocities is the same as in Fig. 2(a), and the deeper colors of right panels show that the velocity is higher for the higher Ra values. The flow velocity with no imposed magnetic field increases in proportion to the square root of $\mathrm{Ra}$ as shown previously [22]. The intensity of the magnetic field required for the suppression of the oscillation depends on Ra. With $\mathrm{Ra}=3.7 \times 10^{4}$, the effect of the magnetic field in reducing the oscillation is observed from $B_{h}=2.6 \mathrm{mT}$, and oscillation is absent at $12.9 \mathrm{mT}$. For $\mathrm{Ra}=1.3 \times 10^{5}$, there is no effect of the magnetic field up to $B_{h}=5.0 \mathrm{mT}$. At this Ra, the oscillation amplitude starts to decrease at $B_{h}=7.5 \mathrm{mT}$, and oscillation is absent at $B_{h}=17.5 \mathrm{mT}$. These data indicate that a stronger magnetic field is necessary to suppress the oscillation of the roll structure with higher Ra values.

Figure 5 shows a time series of the temperatures recorded by the ch3 thermistor probe at Rayleigh numbers ( $\mathrm{Ra}$ ) 3.7 $\times 10^{4}$ and $1.3 \times 10^{5}$. These are the results of the simultaneous measurements with the flow velocities shown in Fig. 


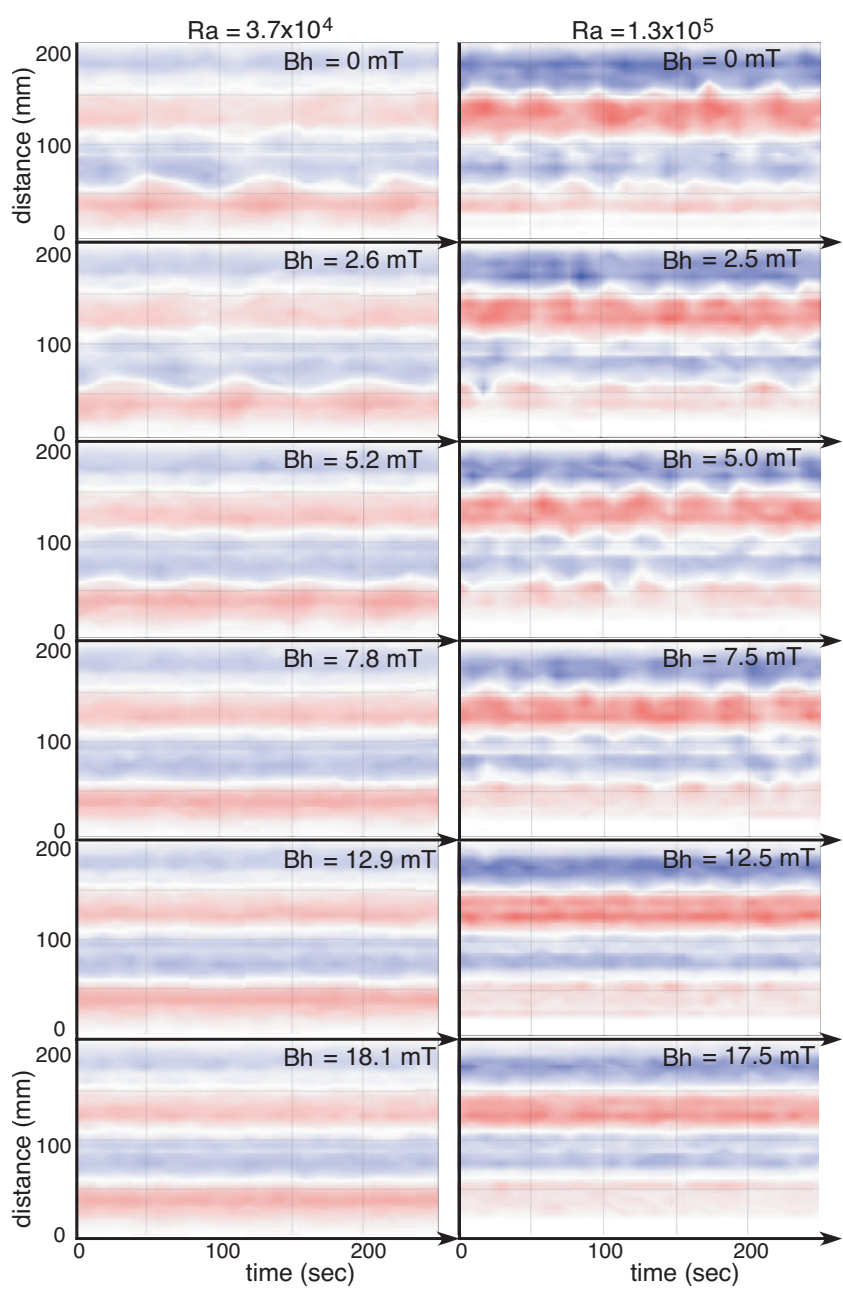

FIG. 4. (Color) Flow patterns observed by UVP for $\mathrm{Ra}=3.7$ $\times 10^{4}$ (left) and $1.3 \times 10^{5}$ (right), with different strengths of the horizontal magnetic fields. The color scale in the velocity graph is the same as in Fig. 2(a). With $\mathrm{Ra}=3.7 \times 10^{4}$, the width of the movement of the central downwelling position decreases from $B_{h}$ $=2.6 \mathrm{mT}$, and oscillation stops at $B_{h}=12.9 \mathrm{mT}$. For $\mathrm{Ra}=1.3$ $\times 10^{5}$, it decreases from $B_{h}=7.5 \mathrm{mT}$, and oscillation stops at $B_{h}$ $=17.5 \mathrm{mT}$.

4. The increase in Ra is achieved by changing the temperature difference between the top and the bottom plates of the convection vessel, and the amplitude of the temperature fluctuations becomes larger for higher Ra values. The graphs are arranged with the applied magnetic field increasing from the top to the bottom for each Ra condition, with the $B_{h}$ value indicated on the right; the " $1 \mathrm{~K}$ " bars at the left indicate the temperature variations. Comparing the temperature time series with the velocity data in Fig. 4 showed that values of $B_{h}$ necessary to reduce and to stop the temperature fluctuations (Fig. 5) are consistent with those for the velocity oscillations (Fig. 4).

To investigate the effect of the magnetic field, further analysis of the velocity and the temperature data was carried out. The flow behavior measured by UVP can be characterized by the oscillation range and a representative velocity. For the oscillation range of the roll structure, the width of movement range of the downwelling position between the
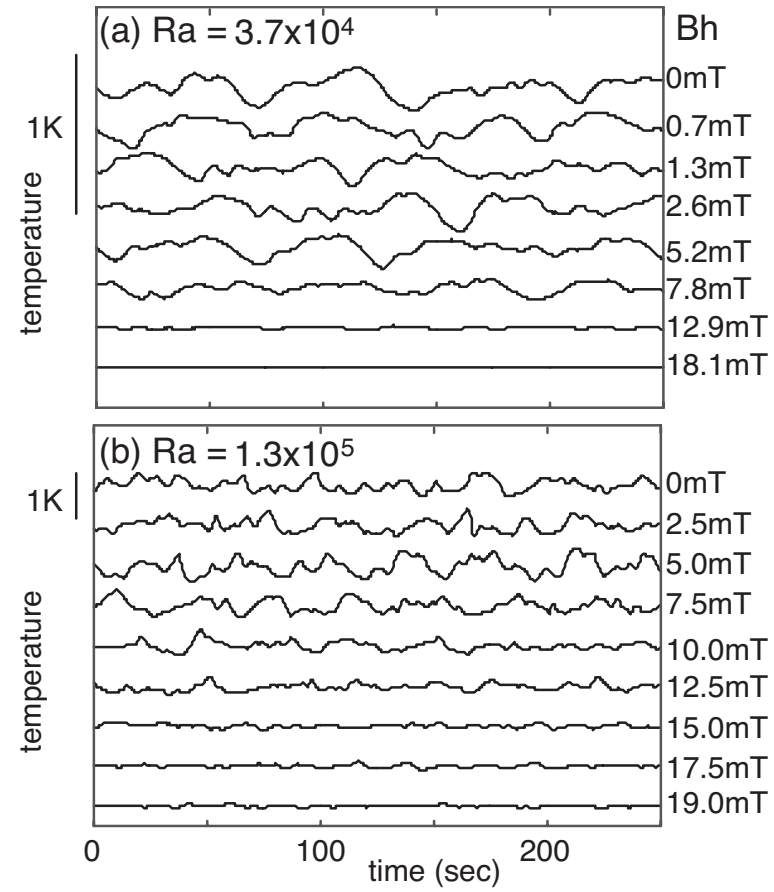

FIG. 5. Time series of temperature fluctuations at the ch3 thermistor probe for Rayleigh numbers (a) $3.7 \times 10^{4}$ and (b) $1.3 \times 10^{5}$. The intensities of the magnetic field are shown at the right. The $1 \mathrm{~K}$ bars on the left are scale bars for the temperature. The amplitude of the temperature fluctuations decreases with the increases in $B_{h}$.

second and the third rolls was used. The representative velocity was the maximum velocity observed in the third roll during each of the magnetic fields. Figure 6(a) shows the width of movement range at the central downwelling position as a function of the intensity of the applied magnetic field $B_{h}$, for the six Ra values investigated here. The oscillation width decreases with the increases in $B_{h}$, but there are differences among the $\mathrm{Ra}$ numbers. For $\mathrm{Ra}=9.3 \times 10^{3}, 1.9$ $\times 10^{4}$, and $3.7 \times 10^{4}$, there are clearly observable decreases above $2.6 \mathrm{mT}$; for $\mathrm{Ra}=7.1 \times 10^{4}$ and $1.3 \times 10^{5}$, the decreases start at $5.2 \mathrm{mT}$; and with $\mathrm{Ra}=3.5 \times 10^{5}$, the decrease starts at $10.0 \mathrm{mT}$. The value of $B_{h}$ to discontinue the oscillation is 7.8 $\mathrm{mT}$ for $\mathrm{Ra}=9.3 \times 10^{3}$, and it increases with increases in $\mathrm{Ra}$, becoming $19.0 \mathrm{mT}$ for $\mathrm{Ra}=1.3 \times 10^{5}$. For the maximum $\mathrm{Ra}$ $\left(3.5 \times 10^{5}\right)$, no region without oscillation was attained even for the maximum intensity of the present coil system $\left(B_{h}\right.$ $=19.0 \mathrm{mT}$ ).

Figure 6(b) shows the maximum velocity in relation to $B_{h}$ for the six Ra values. The flow velocity is mainly controlled by $\mathrm{Ra}$ and increases in proportion to $\mathrm{Ra}^{1 / 2}$ as noted relating to Fig. 4, but there are also slight increases with increases in $B_{h}$. The velocities for $\mathrm{Ra} \leq 7.1 \times 10^{4}$ are very slow ( $\leq 3 \mathrm{~mm} / \mathrm{s}$ for zero magnetic field), and the velocity changes appear smaller than the velocity resolution of UVP $(0.34 \mathrm{~mm} / \mathrm{s})$. For $\mathrm{Ra}=1.3 \times 10^{5}$ and $3.5 \times 10^{5}$, the maximum velocities tend to increase with increases in $B_{h}$. This increase in velocity by UVP measurements may correspond to the decrease in the flow velocity in the horizontal direction normal to the measurement line, and the flow circulating around the roll becomes stronger with the imposition of the two dimensionality of the structure. 


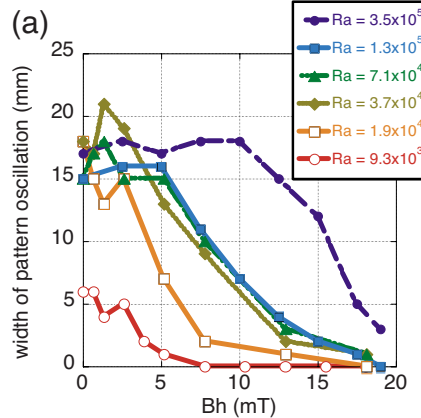

(c)
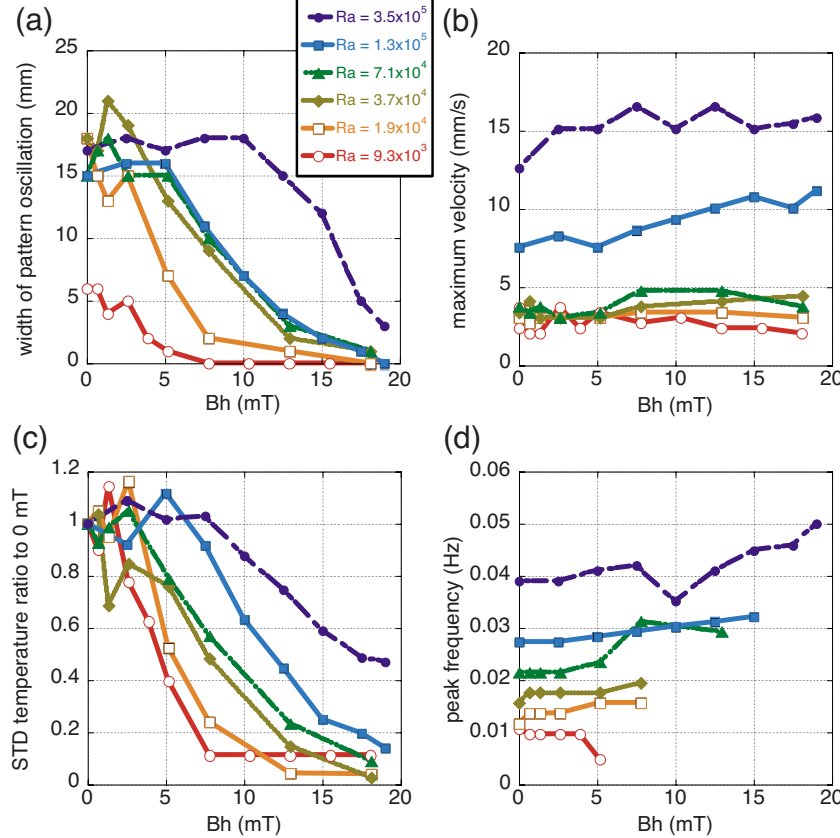

(d)

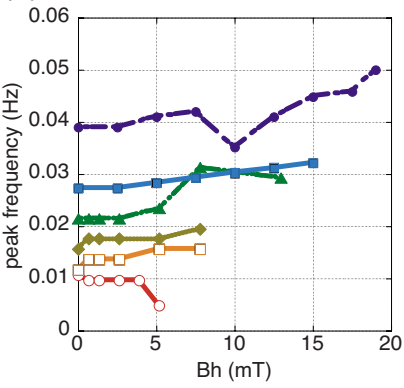

FIG. 6. (Color online) (a) The width of the movement of the central downwelling position in relation to the intensity of applied magnetic field, for six Ra's $9.3 \times 10^{3}, 1.9 \times 10^{4}, 3.7 \times 10^{4}, 7.1$ $\times 10^{4}, 1.3 \times 10^{5}$, and $3.5 \times 10^{5}$. A stronger magnetic field is necessary to stop the oscillation at higher Ra's. (b) The maximum velocity observed in the third roll from left side for these Ra's, slightly increasing with increases in $B_{h}$. (c) The relation between $B_{h}$ and amplitude of the temperature fluctuation expressed by the ratio of standard deviation to $0 \mathrm{mT}$ values. It decreases with increases in $B_{h}$, as the width of the movement of the central downwelling position shown in (a). (d) The relation between $B_{h}$ and the peak frequency, increasing with increases in $B_{h}$ as the maximum velocity shown in (b).

Figure 6(c) shows the normalized standard deviation (STD) of temperature as a function of $B_{h}$, that is, the STD of the temperature fluctuation of each $B_{h}$ is divided by the standard deviation at $0 \mathrm{mT}$. For the two lower Ra values, the standard deviation decreases rapidly with increases in $B_{h}$, and it reaches below the resolution of measurements $(\sim 0.001 \mathrm{~K})$ above $10 \mathrm{mT}$. The rate of decrease for the same $B_{h}$ is smaller for higher $\mathrm{Ra}$, and at $\mathrm{Ra}=3.5 \times 10^{5}$, a $B_{h}$ of 19 $\mathrm{mT}$ reduces to only about half of the reference value. These features of the reduction in temperature STD are similar to the reductions in the width of the pattern oscillation in Fig. 6(a).

Figure 6(d) shows the relation between $B_{h}$ and the peak frequency with the maximum power in the Fourier spectrum of the temperature time series. The peak frequencies are plotted for points that have distinct peaks in the spectra. As also shown in a previous paper [22], the peak frequency is identical to the inverse of the oscillation time of the flow pattern observed in the velocity measurements. Increases in the peak frequency are observed at $B_{h} \geq 5 \mathrm{mT}$ for $\mathrm{Ra} \geq 7.1 \times 10^{4}$. This increase agrees with the increase in the flow velocity with $B_{h}$ in Fig. 6(b), which indicates that the increase in circulation flow velocity mainly causes the frequency variation. Hence, the relation that the oscillation time of the roll structure is comparable to the circulation time appears to be valid even under magnetic fields.

Figure 7 shows the power spectrum densities (PSDs) calculated from the time series of the temperature at four Ra's $3.7 \times 10^{4}, 7.1 \times 10^{4}, 1.3 \times 10^{5}$, and $3.5 \times 10^{5}$. With increases in $B_{h}$, the decreases in temperature fluctuations are observed in these PSDs. This feature is clearly indicated in Fig. 6(c). Additionally, the peak frequency increases slightly with increases in $B_{h}$ for $\mathrm{Ra} \geq 7.1 \times 10^{4}$, in agreement with Fig. 6(d). Temperature PSDs are useful to know whether there are subranges related to turbulence. A thermal energy spectrum model for turbulence in low-Pr fluid by Batchelor et al. [24] predicts a $-5 / 3$ slope for the "inertial-convective" subrange and a $-17 / 3$ slope for the "inertial-conductive" subrange in temperature PSD, and such slopes were reported in [22] for $\mathrm{Ra} \geq 10^{5}$. The gray lines in the PSD graphs indicate slopes of $-5 / 3$ and $-17 / 3$, respectively. For $\mathrm{Ra}=3.7 \times 10^{4}$ there is no region matching these slopes for any $B_{h}$, which indicates that the flow is not turbulent. For higher Ra there are regions that fit these slopes. The slopes of the PSDs for $\mathrm{Ra}=7.1 \times 10^{4}$ with $B_{h} \leq 7.8 \mathrm{mT}$ and for $\mathrm{Ra}=1.3 \times 10^{5}$ with $B_{h} \leq 12.5 \mathrm{mT}$ are consistent with the spectrum model of turbulence. The corner frequency, where the slope changes from $-5 / 3$ to $-17 / 3$, is $\sim 0.14 \mathrm{~Hz}$ for $\mathrm{Ra}=7.1 \times 10^{4}$ and $\sim 0.2 \mathrm{~Hz}$ for $\mathrm{Ra}=1.3 \times 10^{5}$. The PSDs for $\mathrm{Ra}=3.5 \times 10^{5}$ have these slopes for any $B_{h}$ with a corner frequency of $\sim 0.3 \mathrm{~Hz}$, indicating the presence of an inertial-convective subrange of $0.06-0.3$ $\mathrm{Hz}$ and suggesting that the inertial-conductive subrange corresponds to the higher frequency. These PSDs show temperature fluctuation features of turbulence when $\mathrm{Ra}$ is above 7 $\times 10^{4}$ and the flow is oscillatory, and that the corner frequency is little dependent on $B_{h}$. The results indicate that when there is oscillation of the roll-like structure, it generates smaller scale eddies also with an imposed magnetic field. This is consistent with the very small value of the magnetic Prandtl number, suggesting that the magnetic effect is little dependent on the length scale.

\section{DISCUSSION}

Systematic studies of the flow pattern and temperature fluctuations in liquid gallium under various intensities of $B_{h}$ for several $\mathrm{Ra}$ values showed details of the influence of the horizontal magnetic field on the convective flow behavior. The vessel used in the present study has length four times longer than its height with the width being the same as the height. The flow pattern emerging in this vessel without the presence of a magnetic field has a roll structure with the axis basically perpendicular to the long horizontal dimension as also reported in a previous study [22]. The direction of the magnetic field applied in the present experiments was parallel to this roll axis, and hence enforces the roll structure. An effect of the applied magnetic field was clearly observed in the measurements of the velocity profiles. With a magnetic field, the fluctuating components are reduced and the two dimensionality of the roll structure is enhanced; this effect increases with the magnetic field. The rates of reduction in the fluctuations depend on both the $\mathrm{Ra}$ and the intensity of the applied magnetic field as shown in Fig. 6. 

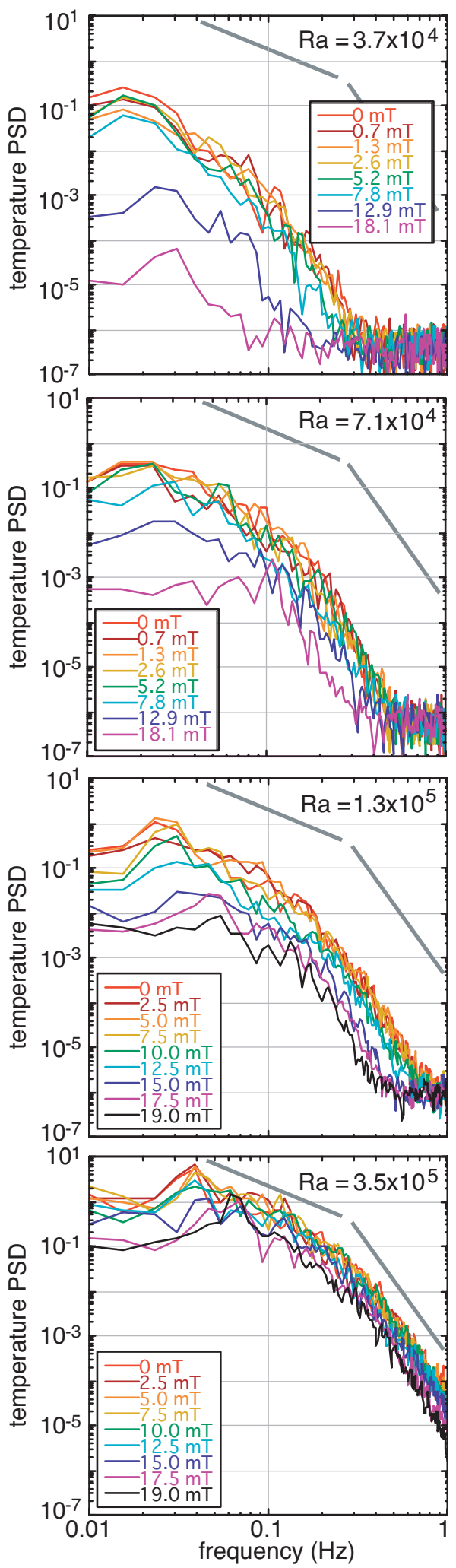

FIG. 7. (Color) PSD graphs calculated from the time series of temperatures for four Ra's. The two gray lines in the graphs indicate slopes of $-5 / 3$ (inertial-convective subrange) and $-17 / 3$ (inertialconductive subrange) predicted by the model of turbulence for low-Pr fluids. The $\mathrm{Ra}=3.7 \times 10^{4}$ field does not show a region with these slopes, while $7.1 \times 10^{4}, 1.3 \times 10^{5}$, and $3.5 \times 10^{5}$ have the regions that fit these slopes well.

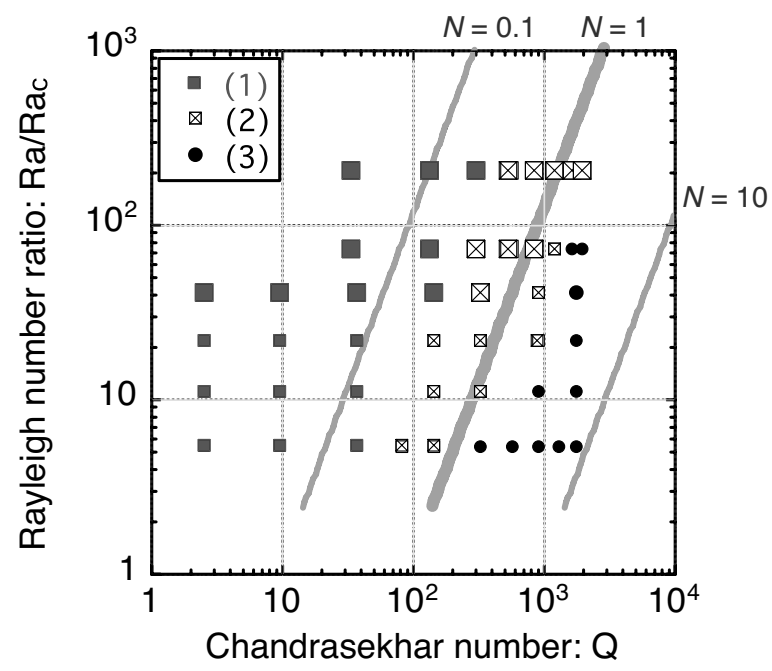

FIG. 8. Regime diagram for the convection behavior plotted as the Chandrasekhar number $Q$ vs the Rayleigh number ratio to the critical $\mathrm{Ra}=1708$. (1) Oscillating roll regime with no effect of a magnetic field, (2) transient regime with decreases in the width of oscillation, and (3) steady two-dimensional roll regime. Larger markers denote points with turbulence determined from the temperature PSDs. Gray lines are isolines of the interaction parameter $N$. The bold gray line $(N=1)$ is the state where the magnitude of the Lorentz force is comparable to the inertial force.

The results shown in Fig. 6 can be summarized as three convection regimes emerging at different $B_{h}$ values: (1) a regime of oscillating rolls with no effect of a magnetic field, (2) a transient regime with decreasing width of the pattern of oscillation, and (3) a regime of steady two-dimensional rolls (no oscillation). The transition in the behaviors is continuous and may be defined as follows: regime (1) the fluctuation width observed by UVP is more than $90 \%$ of that without a magnetic field, regime (2) an intermediate regime, and regime (3) the fluctuation width of less than $3 \mathrm{~mm}$, comparable to twice the spatial resolution of the measurements. Figure 8 shows these convection regimes in relation to the Chandrasekhar number $Q\left(Q=B_{h}^{2} L^{2} \sigma / \rho \nu\right)$ and the ratio of the Rayleigh number to the critical value $\mathrm{Ra} / \mathrm{Ra}_{c}$, in a log-log plot. The critical Ra adopted here is 1708 , the value in a horizontally infinite layer with a no-slip condition at the top and bottom boundaries for the zero magnetic field. The larger markers in regimes (1) and (2) are points showing turbulent behavior as defined by the existence of a $-5 / 3$ slope in the temperature PSDs. These are the points where the oscillating motions of rolls generate and maintain smaller scale of turbulent flows. As Fig. 8 shows, a stronger magnetic field suppresses the fluctuation of convective flows and finally achieves a steady two-dimensional flow. Figure 8 also indicates that the effect of the magnetic field depends on Ra and that a stronger field is necessary to suppress and stop the oscillation of the flow at the higher Ra regime.

The gray lines in Fig. 8 indicate isolines of an interaction parameter $N$, defined by the magnitude ratio of the Lorentz force to the inertial force $\left(N=\sigma B_{h}^{2} L / \rho U\right)$. The three lines are for $N=0.1,1$, and 10: $N=1$ is commonly used to estimate the force balance for low Rm phenomena in magnetohydrodynamics (see, e.g., [1]). In estimating the representative ve- 
locities $U$, the experimentally measured relation of the horizontal flow velocity in this type of vessel without a magnetic field by [22] was used; in this relation, the flow velocity increases as $U L / \kappa=2.4\left(\mathrm{Ra} / \mathrm{Ra}_{c}\right)^{1 / 2}$. This is because $U$ is mainly determined by $\mathrm{Ra}$, and its dependence on $B_{h}$ is relatively small as shown in Fig. 6(b). Substituting this relation into the definition for $N$ gives $\mathrm{Ra} / \mathrm{Ra}_{c}=(\operatorname{Pr} / 2.4)^{2} Q^{2} N^{-2}$, and the gray lines are for $N=0.1,1$, and 10 , with $\operatorname{Pr}=0.025$. The bold gray line $(N=1)$ corresponds to regime (2) where decreases in oscillation width are observed with increases in $B_{h}$. This indicates that the transition Ra from oscillating rolls to steady rolls is related to the intensity of the horizontal magnetic field by $\mathrm{Ra} \sim Q^{2}$. The left area of the bold gray line is the state where the Lorentz force is relatively small and the effect of the magnetic field is weak. The right-hand side is the state where the Lorentz force is relatively large and the flow is strongly affected by the magnetic field. Focusing on the vertical direction, the part above the bold gray line (higher Ra) is the state where buoyancy is dominant and the flow may become turbulent; on the side below the bold gray line (lower Ra), suppression of oscillation by the magnetic field is dominant and laminar flow can be realized. The experiments here observed steady laminar flow structures even at large values of the flow velocity $U$ and $\operatorname{Re} \geq 10^{3}$, when $Q$ is large. This may be explained as an increase in effective viscosity with magnetic fields noted in [11]. The transition to oscillatory convection is related to a critical Reynolds number on the order of 1 . If it is simply assumed that the horizontal magnetic field effectively increases the viscosity as $Q$ times $\nu$, then the effective Re becomes $U L / Q \nu$. The relation $\operatorname{Re}_{e f f}=U L / Q \nu=1$ can be rewritten as $N=1$ from the definition of $Q$, which gives the bold gray line in Fig. 8 .

An experimental study by Libchaber et al. [11] used a vessel with an aspect ratio similar to that in the present study in the Ra range from critical to ten times larger values, with a $Q$ range from 0 to $10^{3}$. Those experiments focused on the behavior of the convection by increasing $\mathrm{Ra}$ at a fixed $Q$ from a steady to a time-dependent flow regime. There the onset point of oscillatory convection for various $Q$ 's and further bifurcations of the oscillation were reported. Bifurcations were observed to occur with slight increases in $\mathrm{Ra}$ around $\mathrm{Ra} / \mathrm{Ra}_{c}=6.7$ when $Q=400$, something that was not observed in the present study. The steps in $Q$ and $\mathrm{Ra}$ are relatively few in the present experiments, and that may be a reason why bifurcations were not observed here. Moreover, the differences in behavior may arise from the direction of the parameter changes; the increase in $B_{h}$ in the present experiment reduces instability, and the increase in $\mathrm{Ra}$ in their experiments increases instability. Libchaber et al. also reported that the amplitude of temperature fluctuation is smaller and the frequency is higher for larger $Q$ values, something that corresponds to the features of regime (2) in the present study.

A horizontal magnetic field does not delay the onset of thermal convection between infinite horizontal planes, but it raises the critical $\mathrm{Ra}$ in a horizontally finite plane because of the existence of sidewall Hartmann layers [16]. The stability analysis by Burr and Müller [16] showed that this effect is more dominant in narrower vessels. In the present experiment, with the side scale of the vessel equal to the height, the instability curve indicates that the increase in critical $\mathrm{Ra}$ is about a factor of 2 for $B_{h}=19 \mathrm{mT}$ and asymptotically $\mathrm{Ra}_{c}$ $\sim Q$. The predicted critical $\mathrm{Ra}$ for this vessel is below the ranges of $\mathrm{Ra}$ and $Q$ in the present experiment, which is consistent with the convection that is observed in all cases here. This assures that in the present experiment the transition from oscillatory to steady convection has a stronger dependence on $Q\left(\mathrm{Ra} \sim Q^{2}\right)$ than the increase in critical Ra with $Q$. The experiments in [16] were carried out using a larger aspect ratio vessel than the present study, with higher $Q$ values $\left(\sim 10^{6}\right)$. The flow pattern in that vessel may be isotropic when $Q$ is small; but with increases in $Q$, many of the features shown by the temperature measurement there were also observed in this study. Burr and Müller reported decreases in temperature fluctuations, increases in the anisotropy of the flow direction, and increases in the Nusselt number, with the increase in $Q$. All of these indicate a damping of the fluctuations and a suppression of turbulence, with restrictions on the direction of the flow. The present results used direct measurements of velocity profiles to establish these features in relation to the global behavior of the flow.

\section{CONCLUDING REMARKS}

The results here report the behavior of Rayleigh-Bénard convection in liquid gallium under the influence of a horizontal magnetic field. The flow pattern was visualized by the measurement of velocity profiles and showed a suppression of oscillatory motion of the two-dimensional roll-like structure with increases in the intensity of the applied magnetic fields. A reduction in temperature fluctuations accompanied this suppression of oscillation. The flow behavior can be classified into three regimes as oscillatory, transient, and steady, expressed by $\mathrm{Ra}$ and $Q$. The transient regime is explained by the relation $N=1$, the magnitude of the Lorentz force comparable to the inertial force, and this indicates that the transition Ra from oscillatory to steady flow is proportional to the square of $Q$. Turbulent behavior characterized by the existence of an inertial-convective subrange in temperature PSDs was observed in the range $\mathrm{Ra} \geq 7 \times 10^{4}$, when the convective motion was oscillatory.

\section{ACKNOWLEDGMENTS}

This work was supported by Grant-in-Aid for Scientific Research No. 21244071 from the Ministry of Education, Culture, Sports, Science and Technology, Japan. The authors express thanks for this support. 
[1] P. A. Davidson, An Introduction to Magnetohydrodynamics (Cambridge University Press, Cambridge, England, 2001).

[2] T. Alboussière, J. P. Garandet, and R. Moreau, J. Fluid Mech. 253, 545 (1993).

[3] L. Davoust, M. D. Cowley, R. Moreau, and R. Bolcato, J. Fluid Mech. 400, 59 (1999).

[4] B. Hof, A. Juel, and T. Mullin, J. Fluid Mech. 545, 193 (2005).

[5] B. Xu, B. Q. Li, and D. E. Stock, Int. J. Heat Mass Transfer 49, 2009 (2006).

[6] S. Chandrasekhar, Hydrodynamic and Hydromagnetic Stability (Oxford University Press, New York, 1961).

[7] F. H. Busse, J. Fluid Mech. 52, 97 (1972).

[8] R. M. Clever and F. H. Busse, J. Fluid Mech. 65, 625 (1974).

[9] F. H. Busse and R. M. Clever, Phys. Fluids 25, 931 (1982).

[10] F. H. Busse and R. M. Clever, Phys. Rev. A 40, 1954 (1989).

[11] A. Libchaber, S. Fauve, and C. Laroche, Physica D 7, 73 (1983).

[12] S. Fauve, C. Laroche, A. Libchaber, and B. Perrin, Phys. Rev. Lett. 52, 1774 (1984).
[13] A. Chiffaudel, B. Perrin, and S. Fauve, Phys. Rev. A 39, 2761 (1989).

[14] J. M. Aurnou and P. L. Olson, J. Fluid Mech. 430, 283 (2001).

[15] U. Burr and U. Müller, Phys. Fluids 13, 3247 (2001).

[16] U. Burr and U. Müller, J. Fluid Mech. 453, 345 (2002).

[17] O. Andreev, A. Thess, and C. Haberstroh, Phys. Fluids 15, 3886 (2003).

[18] Y. Takeda, JSME Int. J., Ser. B 38, 8 (1995).

[19] T. Mashiko, Y. Tsuji, T. Mizuno, and M. Sano, Phys. Rev. E 69, 036306 (2004).

[20] Y. Tsuji, T. Mizuno, T. Mashiko, and M. Sano, Phys. Rev. Lett. 94, 034501 (2005).

[21] Y. Tasaka, Y. Takeda, and T. Yanagisawa, Flow Meas. Instrum. 19, 131 (2008).

[22] T. Yanagisawa, Y. Yamagishi, Y. Hamano, Y. Tasaka, M. Yoshida, K. Yano, and Y. Takeda, Phys. Rev. E 82, 016320 (2010).

[23] K. Okada and H. Ozoe, J. Heat Transfer 114, 107 (1992).

[24] G. K. Batchelor, I. D. Howells, and A. A. Townsend, J. Fluid Mech. 5, 134 (1959). 